\title{
CNV-P: A machine-learning framework for predicting high confident copy number variations
}

\author{
Taifu Wang ${ }^{\text {Equal first author, } 1}$, Jinghua Sun ${ }^{\text {Equal first author, } 1,2}$, Xiuqing Zhang ${ }^{1,2,3}$, Wen-Jing Wang ${ }^{\text {Corresp., } 1}$, Qing Zhou ${ }^{\text {Corresp. } 1}$ \\ 1 BGI-Shenzhen, Shenzhen 518083, China \\ 2 College of Life Sciences, University of Chinese Academy of Sciences, Beijing 100049, China \\ 3 Guangdong Enterprise Key Laboratory of Human Disease Genomics, Beishan Industrial Zone, Shenzhen, 518083, China \\ Corresponding Authors: Wen-Jing Wang, Qing Zhou \\ Email address: wangwenjing@genomics.cn, zhouqing1@genomics.cn
}

Background: Copy-number variants (CNVs) have been recognized as one of the major causes of genetic disorders. Reliable detection of CNVs from genome sequencing data has been a strong demand for disease research. However, current software for detecting CNVs has high false-positive rates, which needs further improvement.

Methods: Here, we proposed a novel and post-processing approach for CNVs prediction (CNV-P), a machine-learning framework that could efficiently remove false-positive fragments from results of CNVs detecting tools. A series of CNVs signals such as read depth (RD), split reads (SR) and read pair (RP) around the putative CNV fragments were defined as features to train a classifier.

Results: The prediction results on several real biological datasets showed that our models could accurately classify the CNVs at over $90 \%$ precision rate and $85 \%$ recall rate, which greatly improves the performance of state-of-the-art algorithms. Furthermore, our results indicate that CNV-P is robust to different sizes of CNVs and the platforms of sequencing.

Conclusions: Our framework for classifying high-confident CNVs could improve both basic research and clinical diagnosis of genetic diseases. 


\section{CNV-P: A machine-learning framework for predicting}

\section{2 high confident copy number variations}

3

4 Taifu Wang $^{1^{*}}$, Jinghua Sun ${ }^{1,2^{*}}$, Xiuqing Zhang ${ }^{1,2,3}$, Wen-Jing Wang ${ }^{1 \#}$, Qing Zhou ${ }^{1 \#}$

5

$6 \quad{ }^{1}$ BGI-Shenzhen, Shenzhen 518083, China

$7 \quad{ }^{2}$ College of Life Sciences, University of Chinese Academy of Sciences, Beijing 100049, China

$8{ }^{3}$ Guangdong Enterprise Key Laboratory of Human Disease Genomics, Beishan Industrial Zone,

9 Shenzhen, 518083, China

10

11 Corresponding Author:

12 Qing Zhou ${ }^{1}$

13 China National GenBank, Jinsha Road, Shenzhen, Guangdong, 518120, China

14 Email address: zhouqing1@genomics.cn 
15

16

17

18

19

20

21

22

23

24

25

26

27

28

29

30

31

32

33

34

35

36

37

38

39

40

41

42

43

44

45

46

47

48

\section{Abstract}

Background: Copy-number variants (CNVs) have been recognized as one of the major causes of genetic disorders. Reliable detection of CNVs from genome sequencing data has been a strong demand for disease research. However, current software for detecting CNVs has high falsepositive rates, which needs further improvement.

Methods: Here, we proposed a novel and post-processing approach for CNVs prediction (CNV$\mathrm{P}$ ), a machine-learning framework that could efficiently remove false-positive fragments from results of CNVs detecting tools. A series of CNVs signals such as read depth (RD), split reads (SR) and read pair (RP) around the putative CNV fragments were defined as features to train a classifier.

Results: The prediction results on several real biological datasets showed that our models could accurately classify the CNVs at over $90 \%$ precision rate and $85 \%$ recall rate, which greatly improves the performance of state-of-the-art algorithms. Furthermore, our results indicate that CNV-P is robust to different sizes of CNVs, as well as the platforms of sequencing.

Conclusions: Our framework for classifying high-confident CNVs could improve both basic research and clinical diagnosis of genetic diseases.

\section{Introduction}

Copy number variations ( $\mathrm{CNVs}$ ) are one of the genetic variations and sequence polymorphisms that widely exist in the human genome. Research shows that CNVs are closely related to the pathogenesis and development of many human diseases such as autism, Parkinson and other neurological diseases (Hollox et al. 2008; Pankratz et al. 2011; Rosenfeld et al. 2010; Sebat et al. 2007). Therefore, accurate detection of CNVs is essential for the diagnosis and research of such diseases.

With the rapid development of high-throughput sequencing technology, genomic sequencing-based technology for CNVs detection has gradually become a leading method owing to its high speed, high resolution, and high repeatability. Many sequencing-based CNVs detection methods have been proposed (Kosugi et al. 2019; Pirooznia et al. 2015; Zhao et al. 2013). Typical CNVs detection approaches mainly utilize three signatures to detect CNVs: read depth (RD), read pairs (RP), split read (SR) (Pirooznia et al. 2015). RD means the number of reads that encompass or overlap CNVs. For example, a deletion indicates a decrease in the average depth of this area. RP refers to the distribution of the insert size of the sequenced library. If the mapping distance of read pairs significantly deviates from the average value of the sequencing library, such discordant alignment features herald the occurrence of CNVs. SR 
49 indicates the split (soft-clipped) alignment features of reads that span CNVs. The initial

50 strategies for detecting CNVs mainly focused on one of these features (Abyzov et al. 2011; Chen

51 et al. 2009; Medvedev et al. 2010). Most of the approaches have high false-positive rates because

52 of the noises of sequencing data, such as sequencing error and artificial chimeric reads.

53 Ambiguous mapping of reads from repeat- or duplication-rich regions also decreases the

54 accuracy of CNVs (Kosugi et al. 2019; Teo et al. 2012). Consequently, tools integrating multiple 55 features to detect CNVs have been gradually developed (Bartenhagen \& Dugas 2016; Layer et

56 al. 2014; Rausch et al. 2012), while their performance still needs be further modified (Kosugi et 57 al. 2019).

58 To identify high confident CNVs, a commonly used strategy is setting a cutoff value or 59 applying various statistical distributions to filter fragments. This strategy greatly depends on the 60 expertise of researchers and their subjective assumptions about the analyzed data. Another 61 strategy uses the intersection of CNVs generated by two or more algorithms. However, due to various $\mathrm{CNV}$-property-dependent and library-property-dependent features used by different

63 detection methods, they usually provide inconsonant results. Thus, a large number of potentially true CNVs could be discarded. Additionally, some tools, such as MetaSV(Mohiyuddin et al. 2015), Parliament2 (Zarate et al. 2020) and FusorSV (Becker et al. 2018), use the method of integrating and merging CNVs from multiple software. These approaches require output results of several certain tools, usually more than four software, while reanalyzing CNVs using their default methods is impractical and time-consuming. Recently, some software using machine learning model to detect or predict CNVs have been developed, such as GATK-SV

(Werling et al. 2018) and CNV- JACG (Zhuang et al. 2020), it is still necessary to develop more accurate tools.

Here, we developed a machine-learning framework for CNVs prediction (CNV-P), aiming to accurately predict CNVs from the results of present software. CNV-P collected three aforementioned signatures (RD, RP and SR) and other information of the putative CNVs. The results of our model on real data demonstrate that CNV-P greatly improves the performance of state-of-the-art algorithms

\section{Materials \& Methods}

\section{Data download and preprocessing}

The gold-standard sets of CNVs from 9 individuals (NA19238, NA19239, NA19240, HG00512, HG00513, HG00514, HG00731, HG00732, HG00733) were downloaded from Chaisson et al (Chaisson et al. 2019). The whole genome sequencing (WGS) data ( 30x) of 
83 these 9 individuals were downloaded from the National Center for Biotechnology Information 84 (NCBI) with an accession number SRP159517 (Table S1, S2). For external validation samples, 85 the sequencing data of NA12878 and HG002 were also downloaded from NCBI with accession 86 numbers SRP159517 and SRP047086 respectively. The gold-standard CNVs of NA12878 were 87 generated by three data sets: the Database of Genomic Variants

88 (http://dgv.tcag.ca/dgv/app/home?ref=GRCh37/hg19) (R. et al. 2013), the 1000 Genomes Project 89 phaseIII (https://ftp.ncbi.nih.gov/1000genomes/ftp/phase3/integrated sv map) (Sudmant et al. 90 2015), and the CNVs of PacBio data from Pendleton, M. et al (Pendleton et al. 2015). The gold91 standard CNVs of HG002 were downloaded from Zook, J.M., et al (Zook et al. 2019).

For the above gold-standard sets, we excluded other types of CNVs except for deletion and duplication, removed CNVs shorter than 100bp and merged fragments with over $80 \%$ reciprocal overlaps. On average, each sample had more than 10,000 CNVs after processing (Table S1). For WGS data, the clean reads after removing adapter and filtering low-quality reads were aligned to the human genome reference (hg19) with bwa (Li 2013) 'mem' command to generate the BAM file. All of these datasets were generated by standard WGS protocol, with libraries of approximate 400bp insert size and average $\sim 30 \mathrm{X}$ coverage (Table $\boldsymbol{S} 2$ ).

100

101

102

103

104

105

106

107

108

109

110

111

112

113

114

115

116

\section{Generate simulated dataset}

We generate random CNVs (range from 100bp to 100kb) based on a copy of human genome (hg19) using mason2 (Holtgrewe 2010). To avoid the same or similar CNVs between training data and test data, we selected fragments on chromosome 1 and chromosome 2 as training samples and CNVs on chromosome 3 and chromosome 4 as testing samples (more details in Table S1). Then, the paired-end sequencing reads (100bp) from the altered genome was simulated by wgsim (Li 2011 ), with an insert size of 500bp and 0.001 base error rate.

\section{Training set and test set}

We chose five common software to obtain the initial sets of CNVs for simulated data and the downloaded sequencing data (deletions and duplications): Lumpy (Layer et al. 2014), Manta (Chen et al. 2015), Pindel (Ye et al. 2009), Delly (Rausch et al. 2012) and breakdancer (Chen et al. 2009). The details of running parameters were shown in the supplemental methods section. The original CNVs were then performed as follows: 1. Removed other types of CNVs except for deletion and duplication. 2. Removed CNVs with $>10$ bp overlapped with $\mathrm{N}$ region of human genome (download from http://genome.ucsc.edu/). 3. Merged CNVs with $\geq 80 \%$ reciprocal overlaps and kept the union part of fragments. 4. Removed CNVs that less than 100bp. Then, we 
117 labeled these treated CNVs as either 'True' or 'False' based on their overlapped part with gold-

118 standard CNVs. CNVs having $\geq 80 \%$ reciprocal overlap with the gold-standard CNVs in

119 simulated data were labeled as "True" and the cutoff was set to $\geq 50 \%$ for sequencing data. We

120 then selected data of 6 individuals as a training set and the other 3 samples as a test set, including

121 two dependent validation datasets (more details in Table S1, S2).

122

\section{Feature extraction}

We chose commonly used signals by detection tools as features in our training model, such as read depth, information of paired and spited read, mapping quality and GC content of CNVs, as well as all these features around CNV's boundaries (Table S3). Training features were obtained from the alignment results (Fig. 1) in BAM format, which was generated by a read aligner that supports partial read alignments, such as BWA-MEM (Li 2013). For read depthbased and GC content-based features, we computed the read depth and GC rate of three regions: 500 bp upstream and downstream of the left breakpoint $\boldsymbol{L}_{\mathbf{b} 1 \mathbf{k}}, 500$ bp upstream and downstream of the right breakpoint $\boldsymbol{R}_{\boldsymbol{b} \boldsymbol{k} \boldsymbol{k}}$, and the region from start to end $\boldsymbol{C}_{\text {start-end }}$. Read depth was calculated by total number of aligned bases divided by the length of the region. We then normalized the read depth by the average coverage of entire genome and processed $\log 2$ transformation to eliminate the impact of fluctuations in sequencing depth. GC content was also calculated in these three regions. Thus, using read depth and GC content of the three local regions $\left(\boldsymbol{L}_{\mathbf{b} 1 \mathrm{k}}, \boldsymbol{R}_{\boldsymbol{b} 1 \boldsymbol{k}}\right.$ and $\boldsymbol{C}_{\text {start-end }}$ ), six features were defined. Split-read, read pair and mapping quality were computed for

two regions: $\boldsymbol{L}_{\mathrm{b} 1 \mathrm{k}}$ and $\boldsymbol{R}_{\boldsymbol{b} 1 \boldsymbol{k}}$. Split read-based features were defined as the number of clipped reads within the area $\boldsymbol{L}_{\mathbf{b} 1 \mathrm{k}}$ or $\boldsymbol{R}_{\boldsymbol{b} 1 \boldsymbol{k}}$. Read pair-based features were defined as the number of outlier reads pair within $\boldsymbol{L}_{\mathrm{b} 1 \mathrm{k}}$ or $\boldsymbol{R}_{\boldsymbol{b} 1 \boldsymbol{k}}$. Normally, The insert size of a normal paired-end read should be within $\boldsymbol{m}_{\boldsymbol{i s}} \pm \boldsymbol{n} \boldsymbol{\sigma}_{\boldsymbol{i s}}$, where $\boldsymbol{m}_{\boldsymbol{i s}}$ and $\boldsymbol{\sigma}_{\boldsymbol{i s}}$ are the median and standard deviation of insert size, the size and type of CNVs as training features, since the efficacy of CNVs could vary for different size ranges and types (duplication/deletion).

148

149 Comparison with CNV-JACG, MetaSV and hard cutoff method 
150 We compared the performance of CNV-P with that of CNV-JACG (Zhuang et al. 2020), 151 MetaSV (Mohiyuddin et al. 2015) and hard cutoff method in the same datasets. Since MetaSV 152 currently does not support Delly's output, only four CNV detection tools (Lumpy, Manta, Pindel, 153 and breakdancer) were taken into consideration. CNV-JACG was conducted running with default 154 parameters (details in supplementary methods). MetaSV was carried out with complete mode. 155 For hard cutoff method, we used SR and RP as the evidence to support the existence of CNVs, 156 therefore, the number of SR and RP greater than 2, 5, and 10 were set as hard cutoff to evaluate. 157 SURVIVOR(Jeffares et al. 2017) was used to merge fragments with 80\% overlap after filtering 158 by CNV-P, CNV- JACG, MetaSV and hard cutoff method.

159

160 Methodology evaluation

we calculated the classifier performance on the test dataset in terms of precision and recall

162 (TP: true positive, TN: true negative, FP: false positive, FN: False negative)

163 Precision $=\frac{\mathrm{TP}}{\mathrm{TP}+\mathrm{FP}}$

$164 \quad$ Recall $=\frac{\mathrm{TP}}{\mathrm{TP}+\mathrm{FN}}$

165 F1 score $=\frac{2 * \text { Precision } * \text { Recall }}{\text { Precision }+ \text { Recall }}$

166 Also, we plotted the ROC with the AUC for model evaluation. ROC curves were drawn 167 based on a series of false positive rates (FPR) and true positive rates (TPR).

168

169

Results

170

Study overview

171

In this study, we built a random forest (RF) framework for the CNVs prediction base on

172 both simulated and real datasets (Fig. 1A). Firstly, we identified CNVs using five common tools (Lumpy, Manta, Pindel, Delly and breakdancer). For each set, we removed CNVs with low quality or locating on the $\mathrm{N}$ region of the human genome (details in Methods). Secondly, we labeled CNVs as either "True" or "False" based on a 50\% reciprocal overlap with the goldstandard CNVs in real data and 80\% reciprocal overlap in simulated data respectively (details in

177 Methods). Next, we extracted the signatures around these CNVs such as RD, SR and RP as training features from alignment results (Fig. $1 B$ ).

We then split the data set into a training set and a test set. Based on the training set, we trained a RF classifier to identify CNVs as "true" or "false". We performed 10-repeated 10-fold 
181 cross-validation for optimal parameter selection and used the receiver operating characteristic 182 (ROC) curve to quantify the prediction performance. Next, we evaluated the robustness of our 183 models on test data from multiple aspects, such as sizes of CNVs and platforms of raw 184 sequencing data. We also compared the performance of Support Vector Machine (SVM) and 185 Gradient Boosting classifier (GBC) with our random forest model. Finally, we validated our 186 model on two extra data sets.

187

\section{Performance of CNV-P on a simulated dataset}

We train RF, GBC, and SVM classifiers for CNV prediction based on a simulated dataset (details in Methods). The results show that there was a significant improvement after CNV-P prediction compared with the original CNV results. The precision of CNVs produce by each CNV detection tools improved from $49.58 \%$ to $99 \%$ with almost zero loss recall rate (Fig. $2 A$, $\boldsymbol{B})$. Compared with GBC and SVM classifiers, RF was slightly superior. The RF classifiers for 5 tools achieved comparable performance since an average increasing of F1-score was about 14.55\% for Lumpy, $14.34 \%$ for Manta, $13.84 \%$ for Pindel, $11.10 \%$ for Delly and $16.16 \%$ for breakdancer (Fig. 2C).

197

198

199

200

201

202

203

204

205

206

207

208

209

210

211

212

213

214

\section{Performance of CNV-P on a real dataset}

In this part, we trained RF classifier for the five selected tools respectively based on real samples. The 10-repeated 10-fold cross-validation was performed for optimal parameter selection (Fig. S1). The overall diagnostic ability of each classifier was measured as the area under the receiver operating characteristic curve (AUC) for the test dataset. The highest value of AUC was $97.10 \%$ for the model of Lumpy while the model for Pindel had the smallest value of 93.62\% (Fig. 3A). Each classifier accurately classified the CNVs as either true or false at 91.7695.17\% precision and $87.75-96.54 \%$ recall rate $($ Fig. 3 B). After processing by CNV-P, a large number of false-positive CNVs were removed, and the majority of true CNVs were remained (Fig. 3C).

To dissect the principle of the CNV-P classifier, we assessed the relative importance of each feature for corresponding classifiers. As expected, for all classifiers, read-depth provided the most discriminatory power to make accurate predictions (Fig. S2). However, the second important feature was inconsistent between different classifiers. It was probably due to various detection algorism these tools used.

To evaluate the robustness of CNV-P, we trained each model on various proportions of training data (from $10 \%$ to $90 \%$ in increments of $20 \%$ ). The results showed a steady 
215 improvement in accuracy (precision and recall rate) with an increase in the number of training 216 data (Fig. S3).

217 We further assessed the performance of CNV-P for CNVs of different sizes. We divided 218 CNVs into three sets based on their length: CNV_S (100 bp to $1 \mathrm{~kb}$; bp: base pair, kb: kilobase), 219 CNV_M (1 kb to $100 \mathrm{~kb})$ and CNV_L (>100kb). The overall precisions were greatly improved, 220 comparing with the raw CNVs achieved by the corresponding software (Fig. 3D). We noticed 221 that almost all precision and recall rates of CNV_S and CNV_M were over 90\%, while theses 222 values of CNV_L were slightly lower. These results are probably caused by the insufficient 223 number of CNV_L in our training data.

224 We also profiled the distribution of predicted probability scores for all CNVs within a 225 different size range. Since CNVs with a probability score $>0.5$ were classified as true in our 226 CNV-P prediction results, we found that the threshold of 0.5 distinguished true and false CNVs 227 very well (Fig. S4). Besides, the probability scores could be used as a measurement of 228 confidence for a certain fragment of $\mathrm{CNVs}$, which would provide support evidence in further 229 analysis.

230 Furthermore, we implanted two additional models, GBC and SVM, to train CNV-P 231 classifiers. Comparing the precision and recall values, as well as the result of ROC curve, we 232 found they had comparable performance (Fig. S5). Still, the RF classifier was recommended as 233 the first choice with a slight superiority.

234

\section{Prediction on external data sets}

236 To further evaluate the performance of CNV-P, we implemented our models on two 237 independent WGS datasets of NA12878 and HG002 (Table S1). Since we had proved that 238 increasing the size of training data could improve the accuracy of our model (Fig. S3), the final 239 classifiers were trained on both the training set and test set mentioned above. Consistent with the 240 above results, CNV-P produced the optimal performance with AUCs of 0.89-0.95 in NA12878

241 (Fig. 4A). Most of the false-positive CNVs were removed with a loss of a small number of true 242 positive fragments (Fig $4 \boldsymbol{B}, \boldsymbol{C}$ ). Likewise, our approach had a similar performance on sample 243 HG002 (Fig. 4D-F).

244 We next compared CNV-P with other post-process tools for CNV filtering, including CNV245 JACG and MetaSV. We also included commonly used hard filtering method, setting cut-off of 246 SR and RP number for each CNV. We applied various filtering approaches on NA12878 and 247 HG002, and then evaluated fragments using gold-standard CNVs of these two samples. Our 248 results showed that CNV-P had the highest F1-score among all the post-process methods (Table 249 1). 
250

251

252

253

254

255

256

257

258

259

260

261

262

263

264

265

266

267

268

269

270

271

272

273

274

275

276

277

278

279

280

281

282

283

Besides, we evaluated the performance of our approach in data generated from multiple sequencing platforms. With precision of 91.6-96.8\% and recall rates of 84.1-94\% (Fig. 5), CNVP showed similar performance on sequencing data generated by BGI-500. Moreover, in addition to the trained classifiers for the above five software, we provided extra modules in our approach for training and predicting if CNVs were detected by other tools. These results suggest that our approach is suitable for CNVs generated from multiple sequencing platforms and detecting software.

\section{Discussion}

Detecting CNVs from WGS is error-prone because of short-length reads and libraryproperty-dependent bias [5]. Inflated false positive makes it a big challenge for researchers to identify clinically relevant CNVs, as it is time- and money-consuming to validate a large amount of false positive CNVs. To solve this problem, we develop CNV-P, an effective machinelearning-based framework to acquire high-confident CNVs. Instead of handling the shortcomings of existing methods by developing another detecting algorithm, CNV-P focuses on providing a reliable set of CNVs from existing detection software. We demonstrate that CNV-P can identify a set of high-confidence CNVs with high precision and recall rates. Moreover, CNV-P is robust to the proportion of variants in training sets, size of CNVs and sequencing platforms, indicating the utility of CNV-P in a variety of clinical or research contexts.

Comparing with the conventional method of using hard cutoff, such as a minimum number of supporting reads, to filtering CNV results, CNV-P greatly reduces errors caused by lack of expertise and subjective assumptions. Instead of running default multiple software in advance, CNV-P can make accurate predictions for each tool dependently. In addition to the five commonly used software that we have trained prediction models, we provide an extra module in CNV-P including the function of model training and predicting if CNVs are detected by other tools.

However, our models may have weaker power for large-size CNVs, because there are only a small number of large fragments in our training data. Besides of data from healthy individuals, we believe that great improvement could be made to identify large-size true CNVs in the future when more datasets are accumulated.

\section{Conclusions}

CNV-P is a well-performed machine-learning framework for accurately filtering CNVs. CNV-P framework can be applied on CNVs from various detection methods and sequencing 
284 platforms, making our framework easy to adopt and customize. CNV-P greatly helps to generate 285 a set of high-confident CNVs, benefiting both basic research and clinical diagnosis of genetic 286 diseases.

287

\section{Availability of data and materials}

289 All data generated or analyzed during this study are included in this published article and its 290 supplementary information files.

291 CNV-P is available at https://github.com/wonderfull/CNV-P.

292

\section{Acknowledgements}

294 The authors thank Dr. Jian Guo for constructive comments on this project and Chen Ye for data 295 download and management.

297 References

298 Abyzov A, Urban AE, Snyder M, and Gerstein M. 2011. CNVnator: an approach to discover, 299 genotype, and characterize typical and atypical CNVs from family and population genome 300 sequencing. Genome Res 21:974-984. 10.1101/gr.114876.110

301 Bartenhagen C, and Dugas M. 2016. Robust and exact structural variation detection with paired302 end and soft-clipped alignments: SoftSV compared with eight algorithms. Brief Bioinform 17:51303 62. 10.1093/bib/bbv028

304 Becker T, Lee WP, Leone J, Zhu Q, Zhang C, Liu S, Sargent J, Shanker K, Mil-Homens A, 305 Cerveira E, Ryan M, Cha J, Navarro FCP, Galeev T, Gerstein M, Mills RE, Shin DG, Lee C, and 306 Malhotra A. 2018. FusorSV: an algorithm for optimally combining data from multiple structural 307 variation detection methods. Genome Biol 19:38. 10.1186/s13059-018-1404-6

308 Chaisson MJP, Sanders AD, Zhao X, Malhotra A, Porubsky D, Rausch T, Gardner EJ, 309 Rodriguez OL, Guo L, Collins RL, Fan X, Wen J, Handsaker RE, Fairley S, Kronenberg ZN, 310 Kong X, Hormozdiari F, Lee D, Wenger AM, Hastie AR, Antaki D, Anantharaman T, Audano 311 PA, Brand H, Cantsilieris S, Cao H, Cerveira E, Chen C, Chen X, Chin CS, Chong Z, Chuang 312 NT, Lambert CC, Church DM, Clarke L, Farrell A, Flores J, Galeev T, Gorkin DU, Gujral M, 313 Guryev V, Heaton WH, Korlach J, Kumar S, Kwon JY, Lam ET, Lee JE, Lee J, Lee WP, Lee 
314 SP, Li S, Marks P, Viaud-Martinez K, Meiers S, Munson KM, Navarro FCP, Nelson BJ, Nodzak 315 C, Noor A, Kyriazopoulou-Panagiotopoulou S, Pang AWC, Qiu Y, Rosanio G, Ryan M, Stutz A, 316 Spierings DCJ, Ward A, Welch AE, Xiao M, Xu W, Zhang C, Zhu Q, Zheng-Bradley X, Lowy 317 E, Yakneen S, McCarroll S, Jun G, Ding L, Koh CL, Ren B, Flicek P, Chen K, Gerstein MB, 318 Kwok PY, Lansdorp PM, Marth GT, Sebat J, Shi X, Bashir A, Ye K, Devine SE, Talkowski ME, 319 Mills RE, Marschall T, Korbel JO, Eichler EE, and Lee C. 2019. Multi-platform discovery of 320 haplotype-resolved structural variation in human genomes. Nat Commun 10:1784.

$321 \quad 10.1038 / \mathrm{s} 41467-018-08148-\mathrm{z}$

322 Chen K, Wallis JW, McLellan MD, Larson DE, Kalicki JM, Pohl CS, McGrath SD, Wendl MC, 323 Zhang Q, Locke DP, Shi X, Fulton RS, Ley TJ, Wilson RK, Ding L, and Mardis ER. 2009.

324 BreakDancer: an algorithm for high-resolution mapping of genomic structural variation. Nat 325 Methods 6:677-681. 10.1038/nmeth.1363

326 Chen X, Schulz-Trieglaff O, Shaw R, Barnes B, and Saunders CT. 2015. Manta: Rapid detection 327 of structural variants and indels for germline and cancer sequencing applications. Bioinformatics $328 \quad 32: 1220-1222$.

329 Hollox EJ, Huffmeier U, Zeeuwen PL, Palla R, Lascorz J, Rodijk-Olthuis D, van de Kerkhof PC, 330 Traupe H, de Jongh G, den Heijer M, Reis A, Armour JA, and Schalkwijk J. 2008. Psoriasis is 331 associated with increased beta-defensin genomic copy number. Nat Genet 40:23-25.

$332 \quad 10.1038 /$ ng.2007.48

333 Holtgrewe M. 2010. Mason@ A Read Simulator for Second Generation Sequencing Data.

334 Jeffares DC, Jolly C, Hoti M, Speed D, Shaw L, Rallis C, Balloux F, Dessimoz C, Bahler J, and 335 Sedlazeck FJ. 2017. Transient structural variations have strong effects on quantitative traits and 336 reproductive isolation in fission yeast. Nat Commun 8:14061. 10.1038/ncomms14061

337 Kosugi S, Momozawa Y, Liu X, Terao C, Kubo M, and Kamatani Y. 2019. Comprehensive 338 evaluation of structural variation detection algorithms for whole genome sequencing. Genome 339 Biol 20:117. 10.1186/s13059-019-1720-5

340 Layer RM, Chiang C, Quinlan AR, and Hall IM. 2014. LUMPY: a probabilistic framework for 341 structural variant discovery. Genome Biol 15:R84. 10.1186/gb-2014-15-6-r84

342 Li H. 2011 wgsim - Read simulator for next generation sequencing. Github Repository [online] 343 http://githubcom/lh3/wgsim 
344 Li H. 2013. Aligning sequence reads, clone sequences and assembly contigs with BWA-MEM. 345 arXiv preprint arXiv:13033997.

346 Medvedev P, Fiume M, Dzamba M, Smith T, and Brudno M. 2010. Detecting copy number

347 variation with mated short reads. Genome Res 20:1613-1622. 10.1101/gr.106344.110

348 Mohiyuddin M, Mu JC, Li J, Bani Asadi N, Gerstein MB, Abyzov A, Wong WH, and Lam HY.

349 2015. MetaSV: an accurate and integrative structural-variant caller for next generation

350 sequencing. Bioinformatics 31:2741-2744. 10.1093/bioinformatics/btv204

351 Pankratz N, Dumitriu A, Hetrick KN, Sun M, Latourelle JC, Wilk JB, Halter C, Doheny KF, 352 Gusella JF, Nichols WC, Myers RH, Foroud T, DeStefano AL, Psg P, GenePd Investigators C, 353 and Molecular Genetic L. 2011. Copy number variation in familial Parkinson disease. Plos One 354 6:e20988. 10.1371/journal.pone.0020988

355 Pendleton M, Sebra R, Pang AW, Ummat A, Franzen O, Rausch T, Stutz AM, Stedman W, 356 Anantharaman T, Hastie A, Dai H, Fritz MH, Cao H, Cohain A, Deikus G, Durrett RE, 357 Blanchard SC, Altman R, Chin CS, Guo Y, Paxinos EE, Korbel JO, Darnell RB, McCombie 358 WR, Kwok PY, Mason CE, Schadt EE, and Bashir A. 2015. Assembly and diploid architecture 359 of an individual human genome via single-molecule technologies. Nat Methods 12:780-786.

$360 \quad 10.1038 /$ nmeth.3454

361 Pirooznia M, Goes FS, and Zandi PP. 2015. Whole-genome CNV analysis: advances in 362 computational approaches. Front Genet 6:138. 10.3389/fgene.2015.00138

363 R. MJ, Robert Z, Yuen RKC, Lars F, and Scherer SW. 2013. The Database of Genomic Variants: 364 a curated collection of structural variation in the human genome. Nucleic Acids Research:D1.

365 Rausch T, Zichner T, Schlattl A, Stütz AM, Benes V, and Korbel JO. 2012. DELLY: structural 366 variant discovery by integrated paired-end and split-read analysis. Bioinformatics 28:i333.

367 Rosenfeld JA, Ballif BC, Torchia BS, Sahoo T, Ravnan JB, Schultz R, Lamb A, Bejjani BA, and 368 Shaffer LG. 2010. Copy number variations associated with autism spectrum disorders contribute 369 to a spectrum of neurodevelopmental disorders. Genet Med 12:694-702.

370 10.1097/GIM.0b013e3181f0c5f3

371 Sebat J, Lakshmi B, Malhotra D, Troge J, Lese-Martin C, Walsh T, Yamrom B, Yoon S, 372 Krasnitz A, Kendall J, Leotta A, Pai D, Zhang R, Lee YH, Hicks J, Spence SJ, Lee AT, Puura K, 373 Lehtimaki T, Ledbetter D, Gregersen PK, Bregman J, Sutcliffe JS, Jobanputra V, Chung W,

374 Warburton D, King MC, Skuse D, Geschwind DH, Gilliam TC, Ye K, and Wigler M. 2007. 
375 Strong association of de novo copy number mutations with autism. Science 316:445-449.

376 10.1126/science.1138659

377 Sudmant PH, Rausch T, Gardner EJ, Handsaker RE, Abyzov A, Huddleston J, Zhang Y, Ye K, 378 Jun G, Fritz MH, Konkel MK, Malhotra A, Stutz AM, Shi X, Casale FP, Chen J, Hormozdiari F, 379 Dayama G, Chen K, Malig M, Chaisson MJP, Walter K, Meiers S, Kashin S, Garrison E, Auton 380 A, Lam HYK, Mu XJ, Alkan C, Antaki D, Bae T, Cerveira E, Chines P, Chong Z, Clarke L, Dal 381 E, Ding L, Emery S, Fan X, Gujral M, Kahveci F, Kidd JM, Kong Y, Lameijer EW, McCarthy S, 382 Flicek P, Gibbs RA, Marth G, Mason CE, Menelaou A, Muzny DM, Nelson BJ, Noor A, Parrish 383 NF, Pendleton M, Quitadamo A, Raeder B, Schadt EE, Romanovitch M, Schlattl A, Sebra R, 384 Shabalin AA, Untergasser A, Walker JA, Wang M, Yu F, Zhang C, Zhang J, Zheng-Bradley X, 385 Zhou W, Zichner T, Sebat J, Batzer MA, McCarroll SA, Genomes Project C, Mills RE, Gerstein 386 MB, Bashir A, Stegle O, Devine SE, Lee C, Eichler EE, and Korbel JO. 2015. An integrated map 387 of structural variation in 2,504 human genomes. Nature 526:75-81. 10.1038/nature15394

388 Teo SM, Pawitan Y, Ku CS, Chia KS, and Salim A. 2012. Statistical challenges associated with 389 detecting copy number variations with next-generation sequencing. Bioinformatics 28:2711390 2718. 10.1093/bioinformatics/bts535

391 Werling DM, Brand H, An JY, Stone MR, Zhu L, Glessner JT, Collins RL, Dong S, Layer RM, 392 Markenscoff-Papadimitriou E, Farrell A, Schwartz GB, Wang HZ, Currall BB, Zhao X, Dea J, 393 Duhn C, Erdman CA, Gilson MC, Yadav R, Handsaker RE, Kashin S, Klei L, Mandell JD, 394 Nowakowski TJ, Liu Y, Pochareddy S, Smith

395 L, Walker MF, Waterman MJ, He X, Kriegstein AR, Rubenstein JL, Sestan N, McCarroll SA, N 396 eale BM, Coon H, Willsey AJ, Buxbaum JD, Daly MJ, State MW, Quinlan AR, Marth GT, Roed 397 er K, Devlin B, Talkowski ME, and Sanders SJ. 2018. An analytical framework for whole398 genome sequencing association studies and its implications for autism spetrum disorder. Nat 399 Genet 50:727-736. 10.1038/s41588-018-0107-y

400 Ye K, Schulz MH, Long Q, Apweiler R, and Ning Z. 2009. Pindel: a pattern growth approach to 401 detect break points of large deletions and medium sized insertions from paired-end short reads. 402 Bioinformatics 25:2865-2871. 10.1093/bioinformatics/btp394

403 Zarate S, Carroll A, Mahmoud M, Krasheninina O, Jun G, Salerno WJ, Schatz MC, Boerwinkle 404 E, Gibbs RA, and Sedlazeck FJ. 2020. Parliament2: Accurate structural variant calling at scale. 405 Gigascience 9. 10.1093/gigascience/giaa145 
406 Zhao M, Wang Q, Wang Q, Jia P, and Zhao Z. 2013. Computational tools for copy number 407 variation $(\mathrm{CNV})$ detection using next-generation sequencing data: features and perspectives. Bmc 408 Bioinformatics 14 Suppl 11:S1. 10.1186/1471-2105-14-S11-S1 409 Zhuang X, Ye R, So MT, Lam WY, Karim A, Yu M, Ngo ND, Cherny SS, Tam PK, Garcia410 Barcelo MM, Tang CS, and Sham PC. 2020. A random forest-based framework for genotyping 411 and accuracy assessment of copy number variations. NAR Genom Bioinform 2:lqaa071.

412 10.1093/nargab/lqaa071

413 Zook JM, Hansen NF, Olson ND, Chapman LM, Mullikin JC, Xiao C, Sherry S, Koren S, 414 Phillippy AM, Boutros PC, Sahraeian SME, Huang V, Rouette A, Alexander N, Mason CE, 415 Hajirasouliha I, Ricketts C, Lee J, Tearle R, Fiddes IT, Barrio AM, Wala J, Carroll A, Ghaffari 416 N, Rodriguez OL, Bashir A, Jackman S, Farrell JJ, Wenger AM, Alkan C, Soylev A, Schatz MC, 417 Garg S, Church G, Marschall T, Chen K, Fan X, English AC, Rosenfeld JA, Zhou W, Mills RE, 418 Sage JM, Davis JR, Kaiser MD, Oliver JS, Catalano AP, Chaisson MJ, Spies N, Sedlazeck FJ, 419 and Salit M. 2019. A robust benchmark for germline structural variant detection.

420 bioRxiv:664623. 10.1101/664623 
Figure 1

The study overview of CNV-P

A) The workflow of CNV-P framework classifyng candidate CNVs as True or False. B) The features we used to train supervised machine learning models

A

Read alignments

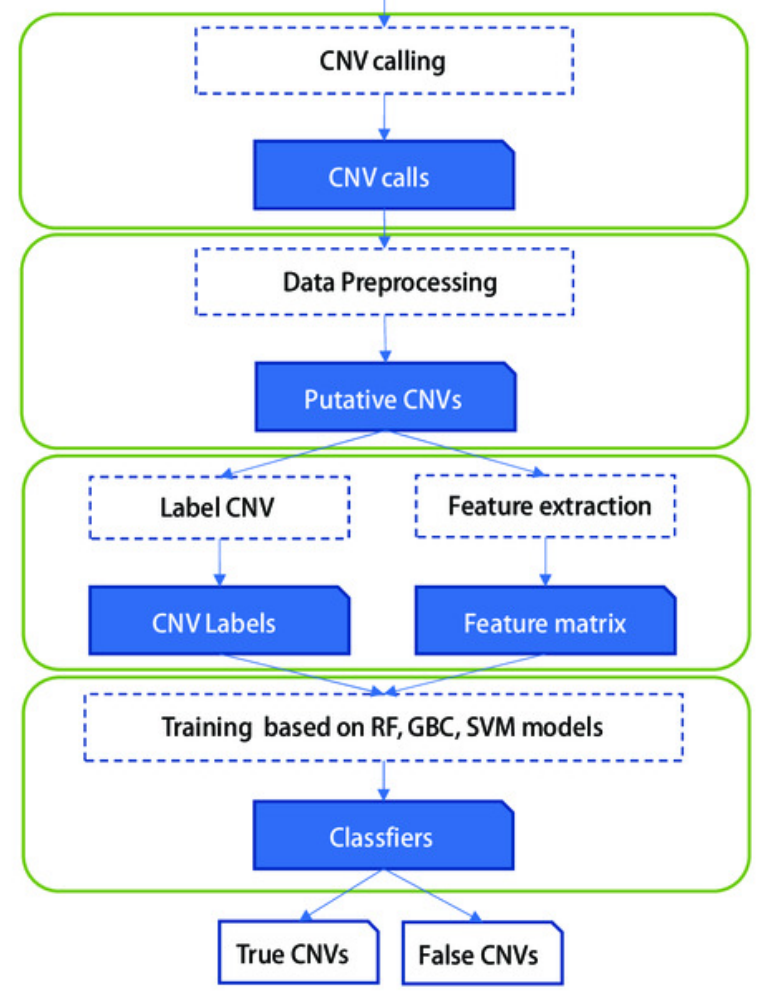

B

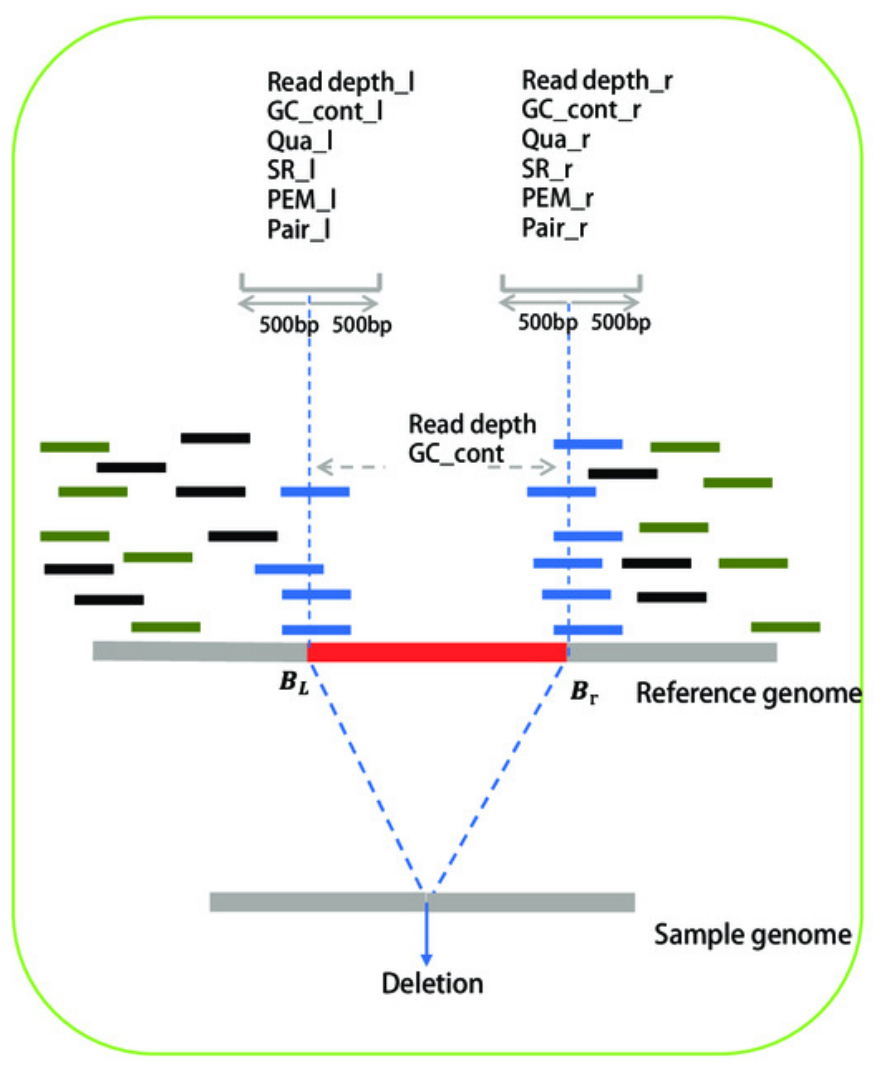


Figure 2

Performance of CNV-P on simulated dataset.

A)The F-score , B) sensitivity, and C) precision over testing simulated dataset.

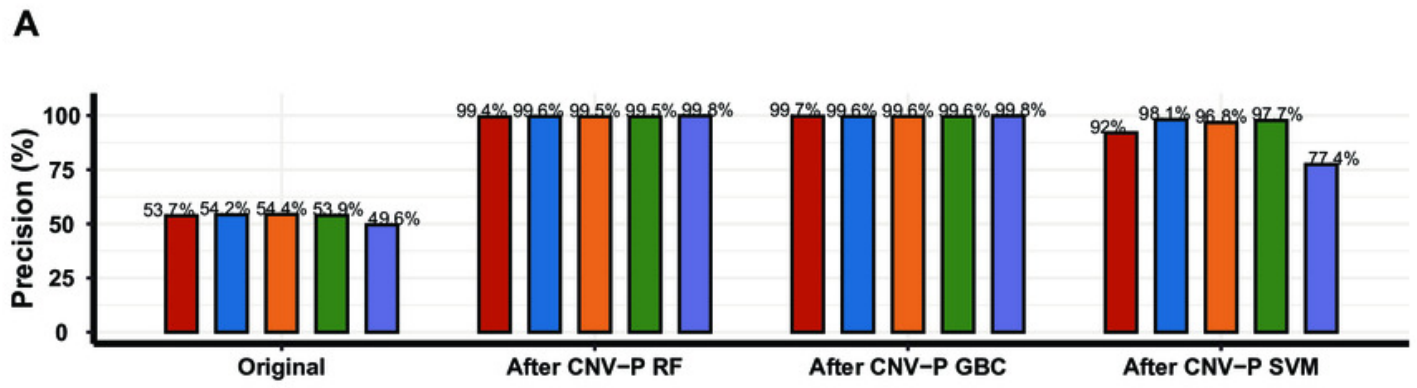

B

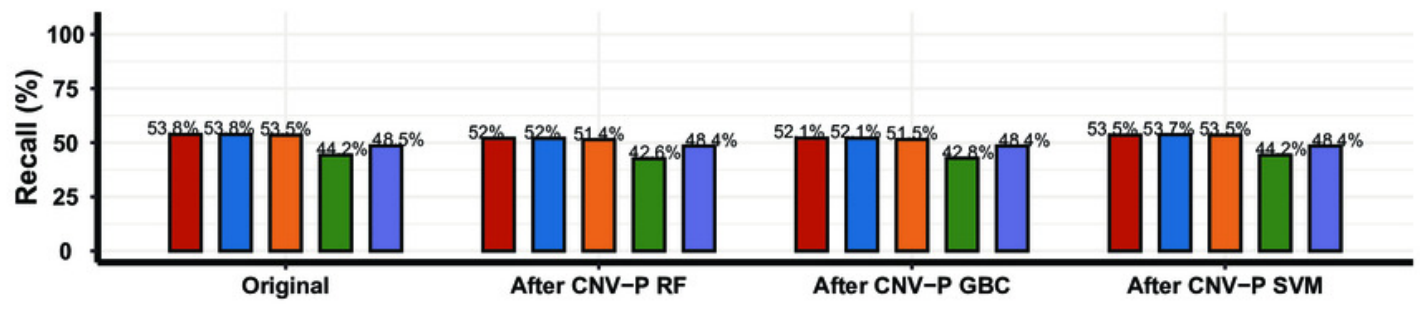

C

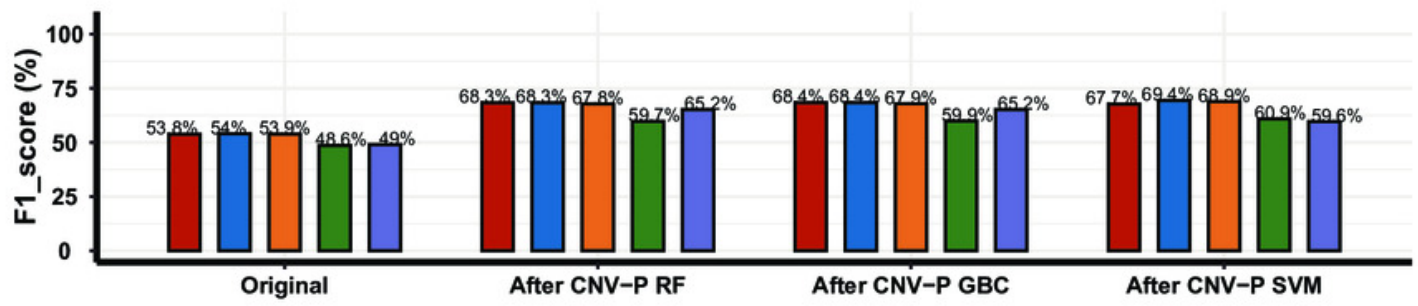

$\square$ Lumpy $\square$ Manta $\square$ Pindel $\square$ Delly $\square$ breakdancer 
Figure 3

Performance of CNV-P on real dataset.

A) Area Under the receiver operating characteristic Curve (AUC) of CNV-P in 3 test datasets.

B) The precise and recall rate of CNV-P. C) The number of CNVs before and after CNV-P

predicting for five commonly used tools. D) The precise and recall rate of CNV-P at different size range of CNVs. CNV_S: 100 bp to $1 \mathrm{~kb}$, CNV_M: $1 \mathrm{~kb}$ to $100 \mathrm{~kb}, \mathrm{CNV}$ L: $>100 \mathrm{~kb}$.

A

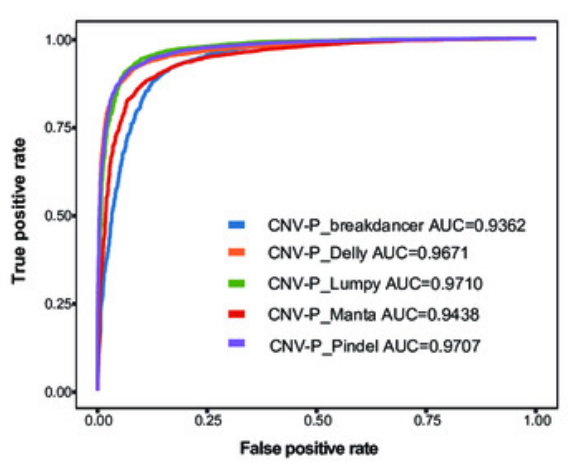

D
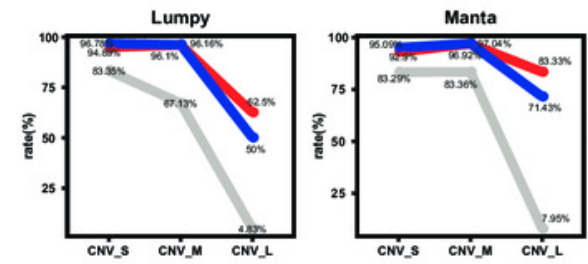

B

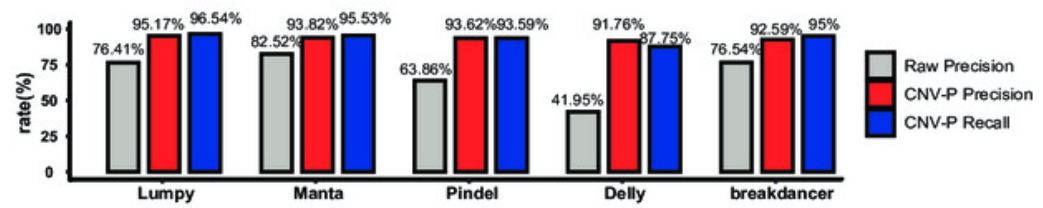

c
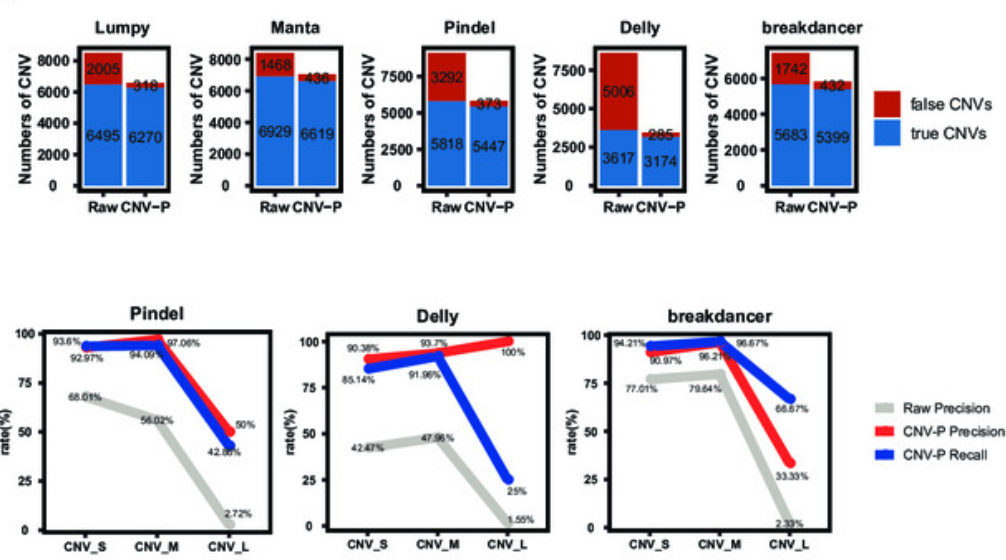

CNV-P Procision

- CNV-PRecall 
Figure 4

Performance of CNV-P on other validation dataset.

CNV-P detects high-confident CNVs with high precision and recall rates on two independent sequencing datasets from NA12878 (A, B, C) and HG002 (D, E, F). (A, D) Receiver operating characteristic (ROC) curves of CNV-P. (B, E) The precision and recall rate of CNV-P; (C, F) The number of classified CNVs by CNV-P from five commonly used tools.

A

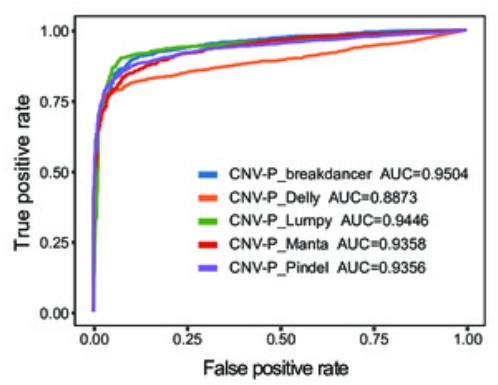

D

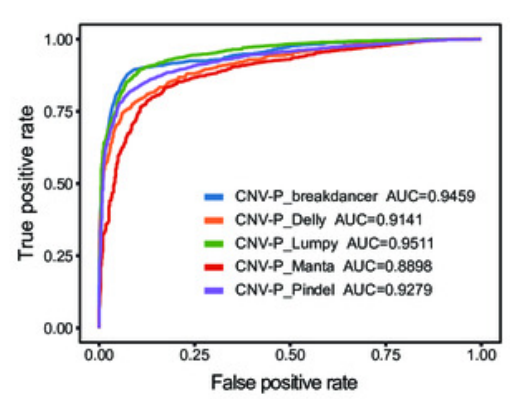

B

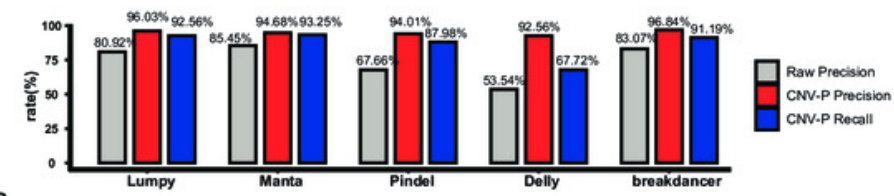

C

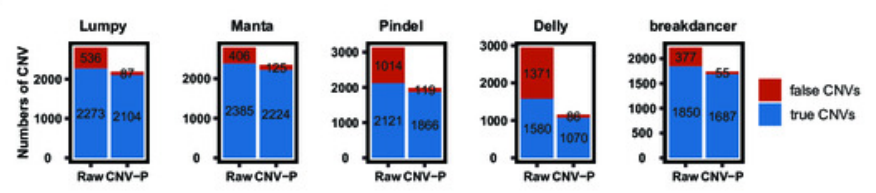

E
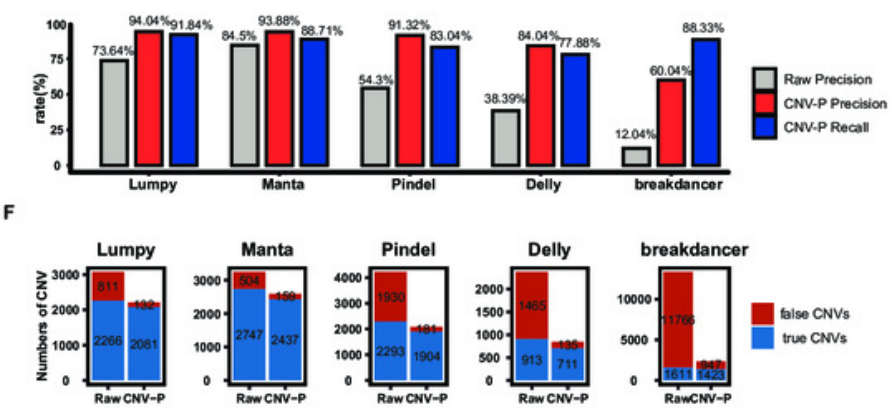

breakdancer 
Figure 5

Performance of CNV-P on different sequencing platform.

The precise and recall rate of CNV-P for sample NA12878 using sequencing data generated from BGI-SEQ500 and Illumina. A) The raw precise results; B) Precise rate; C) Recall rate.

A

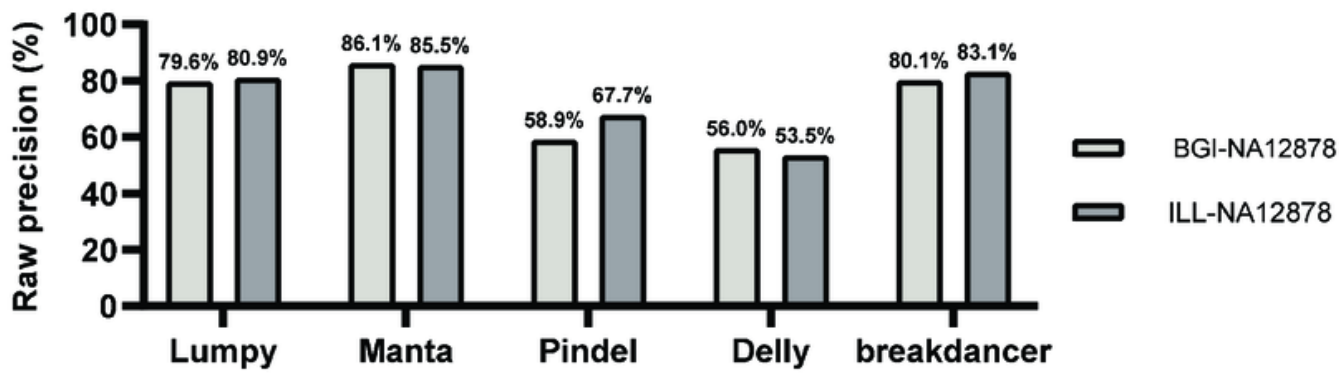

B

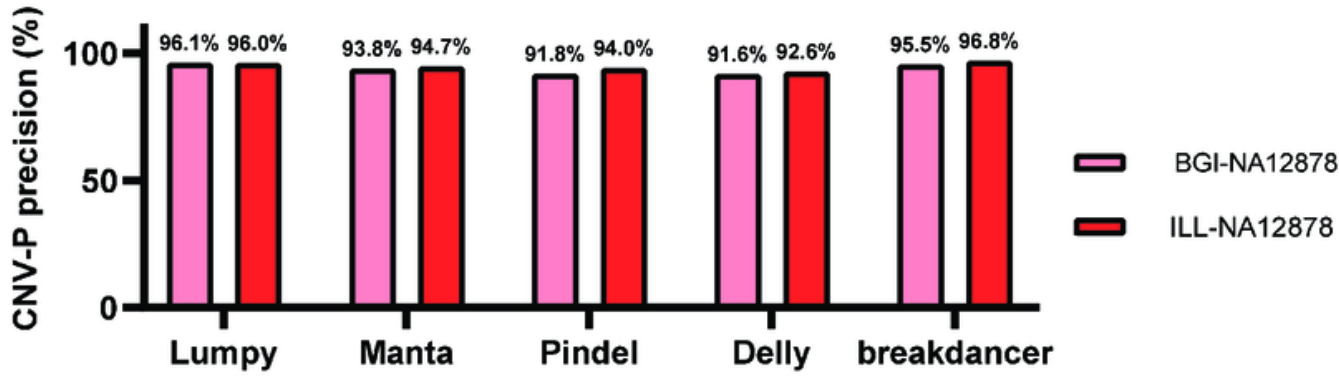

C

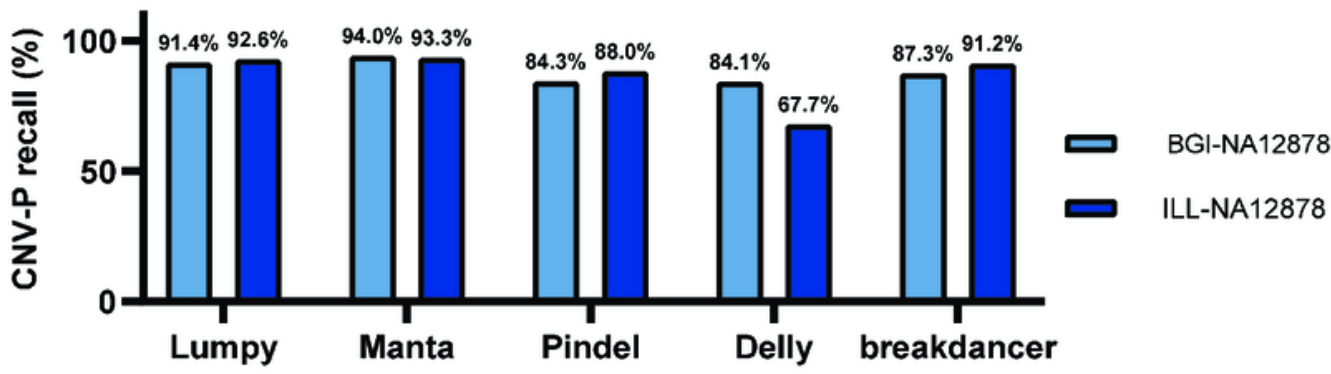




\section{Table $\mathbf{1}$ (on next page)}

Comparison with CNV-JACG, MetaSV and hard cutoff method in NA12878 and HG002 
1 Table 1: Comparison with CNV-JACG, MetaSV and hard cutoff method in NA12878 and HG002.

\begin{tabular}{|l|l|l|l|l|}
\hline Sample & method & precise & recall & F1-score \\
\hline \multirow{5}{*}{ NA12878 } & RAW & 0.6032 & 1.0000 & 0.7525 \\
\cline { 2 - 5 } & Hard_Cutoff_2 & 0.6197 & 0.9792 & 0.7590 \\
\cline { 2 - 5 } & Hard_Cutoff_5 & 0.7145 & 0.8630 & 0.7818 \\
\cline { 2 - 5 } & Hard_Cutoff_10 & 0.7780 & 0.6976 & 0.7356 \\
\cline { 2 - 5 } & CNV-JACG & 0.6828 & 0.7496 & 0.7146 \\
\cline { 2 - 5 } & MetaSV & 0.7094 & 0.8817 & 0.7862 \\
\cline { 2 - 5 } & CNV-P & $\mathbf{0 . 9 0 0 7}$ & $\mathbf{0 . 7 9 7 7}$ & $\mathbf{0 . 8 4 6 1}$ \\
\hline \multirow{5}{*}{ HG002 } & RAW & 0.2054 & 1.0000 & 0.3408 \\
\cline { 2 - 5 } & Hard_Cutoff_2 & 0.4026 & 0.9729 & 0.5695 \\
\cline { 2 - 5 } & Hard_Cutoff_5 & 0.5740 & 0.8653 & 0.6901 \\
\cline { 2 - 5 } & Hard_Cutoff_10 & 0.6642 & 0.7482 & 0.7037 \\
\cline { 2 - 5 } & CNV-JACG & 0.5443 & 0.7076 & 0.6153 \\
\cline { 2 - 5 } & MetaSV & 0.5917 & 0.8274 & 0.6900 \\
\cline { 2 - 5 } & CNV-P & $\mathbf{0 . 7 0 7 8}$ & $\mathbf{0 . 7 5 1 6}$ & $\mathbf{0 . 7 2 9 0}$ \\
\hline
\end{tabular}

2 